\title{
Copy number alterations and epithelial-mesenchymal transition genes in diffuse and intestinal gastric cancers in Mexican patients
}

\author{
Violeta Larios-Serrato ${ }^{1 *}$, José-Darío Martínez-Ezquerro ${ }^{2,3 *}$, Hilda-Alicia Valdez-Salazar ${ }^{4 *}$, \\ Javier Torres ${ }^{4}$, Margarita Camorlinga-Ponce ${ }^{4}$, Patricia Piña-Sánchez ${ }^{5}$, Martha-Eugenia Ruiz- \\ Tachiquín ${ }^{5}+$
}

${ }^{+}$Corresponding author: Martha-Eugenia Ruiz-Tachiquín; mertachiquin@ gmail.com

* Equal contribution

${ }^{1}$ Laboratorio de Biotecnología y Bioinformática Genómica, Escuela Nacional de Ciencias Biológicas (ENCB), Instituto Politécnico Nacional (IPN), Unidad Profesional Lázaro Cárdenas, Prolongación de Carpio y Plan de Ayala S/N. Col Santo Tomás, Alcaldía Miguel Hidalgo, CP 11340, Ciudad de México, México.

${ }^{2}$ Unidad de Investigación Epidemiológica y en Servicios de Salud, Área Envejecimiento (UIESSAE), Centro Médico Nacional Siglo XXI, Instituto Mexicano del Seguro Social (IMSS). Av. Cuauhtémoc 330, Colonia Doctores, Alcaldía Cuauhtémoc, CP 06720, Ciudad de México, México

${ }^{3}$ Centro de Ciencias de la Complejidad (C3), Universidad Nacional Autónoma de México (UNAM), Circuito Centro Cultural S/N, Cd. Universitaria, Alcaldía Coyoacán, CP 04510, Ciudad de México, México

${ }^{4}$ Unidad de Investigación Médica en Enfermedades Infecciosas y Parasitarias (UIMEIP), UMAE-Hospital de Pediatría "Dr. Silvestre Frenk Freund", Centro Médico Nacional Siglo XXI, Instituto Mexicano del Seguro Social (IMSS). Av. Cuauhtémoc 330, Colonia Doctores, Alcaldía Cuauhtémoc, CP 06720, Ciudad de México, México

${ }^{5}$ Unidad de Investigación Médica en Enfermedades Oncológicas (UIMEO), UMAE-Hospital de Oncología, Centro Médico Nacional Siglo XXI, Instituto Mexicano del Seguro Social (IMSS). Av. Cuauhtémoc 330, Colonia Doctores, Alcaldía Cuauhtémoc, CP 06720, Ciudad de México, México

Running title: CNA and EMT genes in gastric cancer in Mexican patients

Keywords: CNA, gastric cancer, high density arrays, EMT 


\title{
Financial support
}

The present study was supported by Fondo de Investigación en Salud-Instituto Mexicano del Seguro Social (Grant numbers: FIS/IMSS/PROT/G16/1573 to M.-E. Ruiz-Tachiquín and FIS/IMSS/PROT/PRIO/13/027 to J. Torres).

\section{Conflict of interest}

The authors declare no potential conflicts of interest.

\begin{abstract}
Gastric cancer (GC) is a malignancy with the highest mortality among diseases of the digestive system worldwide. The study of GC-alterations is crucial to understand tumor biology, to establish important aspects of cancer prognosis and treatment response. Here, we purified DNA and performed whole-genome analysis with high-density arrays in samples from Mexican patients diagnosed with GC: diffuse (DGC) or intestinal (IGC), or non-atrophic gastritis (NAG) samples that served as controls. We identified shared and unique copy number alterations (CNA) between these altered tissues involving key genes and signaling pathways associated with cancer, allowing their molecular distinction and identification of the most relevant molecular functions impacted. When focused on epithelial-mesenchymal transition (EMT) genes, our bioinformatic analysis revealed that the altered network associated with chromosomal alterations included 11 genes shared between DGC, IGC, and NAG, as well as 19 DGC- and 7 IGC-exclusive genes, whose main molecular functions included adhesion, angiogenesis, migration, metastasis, morphogenesis, proliferation, and survival. This study presents the first whole-genome high-density array study in GC from Mexican patients and reveals shared and exclusive CNA-genes in DGC and IGC. In addition, we provide a bioinformatically predicted network focused on CNA-altered genes involved in the EMT, associated with the hallmarks of cancer, as well as precancerous alterations that could lead to gastric cancer.
\end{abstract}

Implications: Molecular signatures of diffuse and intestinal GC, predicted bioinformatically, involve common and distinct CNA-EMT genes related to the hallmarks of cancer that are potential candidates for screening GC biomarkers, including early stages. 


\section{Introduction}

According to Global Cancer Observatory (GCO or GLOBOCAN) statistics, cancer is the leading cause of death in the World with 9.9 million deaths in 2020. This year, cancer had an incidence of $20 \%$ in the Caribbean and South America with high mortality rates (14\%). Worldwide, gastric cancer (GC) is estimated to be the fifth most common cancer in both genders, ranking sixth in new cases with over one million per year and third in mortality (1). In Mexico, according to statistics from the Instituto Nacional de Estadística y Geografía (INEGI), three out of ten cancer deaths in the age group among 30-59 years old were due to cancer of the digestive system. From 2011 to 2016, four out of 10 and three out of 10 cancer deaths in women and men aged 60 years old or more, respectively, resulted from tumors in digestive organs (2).

GC refers to any malignancy originating in the region between the gastroesophageal junction and the pylorus. The World Health Organization and the Lauren classification system (3) have described two types of GC: intestinal and diffuse. The intestinal or differentiated gastric cancer (IGC) is characterized by localized and expansive growth, while diffuse gastric cancer (DGC) has an infiltrating growth pattern, is an undifferentiated adenocarcinoma, and presents dispersed cells with individual or group invasive capacity (4). The development of IGC is preceded by a precancerous process of several years and stages: active chronic gastritis, multifocal atrophic gastritis, complete intestinal metaplasia, incomplete intestinal metaplasia, dysplasia and adenocarcinoma (5). GC has a multifactorial origin: diet, lifestyle, genetics, socioeconomic factors, and it has been observed that $80 \%$ of cases of IGC are associated with previous Helicobacter pylori infection (6,7). GC is characterized by a complex etiology with a set of factors, such as genetic alterations and external factors. However, it has been reported that less than $3 \%$ of GC is due to heredity, and involves hereditary DGC, proximal polyposis of the stomach, and hereditary colorectal cancer not associated with polyposis (6). Regarding molecular pathogenesis, chromosomal instability (aneuploidy, chromosomal translocation, amplification, deletions and loss of heterozygosity), gene fusion and microsatellite instability (hypermethylation of gene repair promoters) are involved (7).

The number of copy alterations (CNA) represent a class of genetic variation that involve cumulative somatic variations, CNA are defined as non-inherited genetic alterations that occur 
in somatic cells (8). These unbalanced structural variants usually contain gains or losses. Its interpretation and the CNA report continue to be a topic of interest in health, and become evident to play an important role in $\mathrm{GC}(9,10)$.

The majority of gastric adenocarcinomas, like many other solid tumors, show defects in the maintenance of genome stability, resulting in DNA CNA that can be analyzed by comparative genomic hybridization (CGH) (11). There is a widespread and common phenomenon among humans and several studies have focused on understanding these genomic alterations that are responsible for cancer and might be used in diagnosis and prognosis (12).

Currently, there are few published studies involving genotyping of GC samples employing high-density microarrays (13-15); however, in those altered chromosomes, gains and losses have a phenotypic impact, and different signaling pathways are involved. The presence of CNA changes the genetic dose and would modify several molecular mechanisms as epithelialmesenchymal transition (EMT), which is the transformation of epithelial to mesenchymal cells and a critical stage for the transition to metastasis (16). Currently, there are more than 1184 genes at the Epithelial-Mesenchymal Transition Gene Database 2.0 (dbEMT 2.0), which are involved in other cancer-related processes such as proliferative signaling, evading growth suppressors, avoiding immune destruction, disabling replicative immortality, tumor-promoting inflammation, inducing angiogenesis, genome instability, mutation, resting cell death, deregulation cellular energetic activity, invasion and cell plasticity (17). EMT includes activation of transcription factors, expression of specific cell-surface proteins, reorganization and expression of cytoskeletal proteins, and production of extracellular matrix (ECM) degrading enzymes (18). EMT has been linked to the progression of cancer and increased stemness of tumors $(18,19)$, and observed in the formation, invasion and metastasis of GC (20,21). Also, a relationship has been established between the presence of CNA and its effect on the expression of EMT genes in different cancer cell lines (22). Reports of CNA events involving Latin American populations are still scant $(23,24)$. In fact, at present there are few studies of GC genotyping by whole-genome high-density microarrays in Mexican patients $(14,15)$. Therefore, our study aimed to determine CNA in DGC and IGC to identify through bioinformatics analyses, the main genes and signaling pathways involving EMT-genes.

\section{Material and Methods}




\section{Samples}

Institutional Review Board approval was obtained for the study origin (Register number: 2008785-001). Clinical data and patient samples were processed under informed consent. All samples were collected over three years (April 2010-May 2013) following standardized endoscopy preservation protocols (25). The histology of the biopsies was assessed independently by two trained pathologists. They assigned the phenotypic diagnosis of diffuse or intestinal tumors and non-atrophic gastritis (NAG) samples. Only samples with identical results were included in the study.

We included 21 patients (5 females and 16 males) with tissue samples that met the quality criteria from DGC $(n=7)$ and IGC $(n=7)$ diagnoses, as well as NAG $(n=7)$ as controls. To identify the most relevant alterations for GC, we focused our analysis on alterations present in at least three patients as an arbitrarily threshold (cut-off $\geq 3$ patients; $\geq 40 \%$ samples).

\section{DNA extraction}

DNA extraction was done with a commercial kit (QIAamp® micro Kit, QIAGEN®) according to the manufacturer's instructions. The extraction was modified to include an initial incubation at $95^{\circ} \mathrm{C}$ for $15 \mathrm{~min}$ followed by $5 \mathrm{~min}$ at room temperature as described previously, before being digested with proteinase $\mathrm{K}$ for three days at $56^{\circ} \mathrm{C}$ in a water-bath adding fresh enzyme at $24 \mathrm{~h}$ intervals (26).

\section{DNA quality assessment and preparation}

The extracted DNA was quantified by spectrophotometry (Nanodrop 2000, Thermo Scientific). Multiplex PCR was done to assess the quality of DNA (Multiplex PCR kit, QIAGEN®) with a set of primers to amplify various regions of the GAPDH gene (27). Products were visualized by electrophoresis (RedGel® Nucleic acid gel stain, Biotium) on a 1\% agarose gel and documented under an ultraviolet light transilluminator system (Syngene, Frederick, MD, USA).

\section{High-density whole-genome microarray analysis}

Samples were analyzed by Affymetrix ${ }^{\circledR}$ CytoScan ${ }^{\mathrm{TM}}$ microarrays according to the manufacturer's protocol beginning with $250 \mathrm{ng}$ DNA, except for the addition of five PCR cycles 
to increase the DNA sample. PCR products (90 micrograms) were fragmented and labeled using the additional PCR.

\section{Copy number processing}

Raw intensity files (.CEL) retrieved from the commercial platform were analyzed using their proprietary software Chromosome Analysis Suite v3.2, using NetAff 33 Libraries based on the construction of the hg19 genome (Feb 2009) as a reference model.

Data processing was based on the segmentation algorithm, where the $\log _{2}$ ratio for each marker was calculated relative to the reference signal profile. To calculate CNV, the data were normalized to baseline reference intensities using the reference model (provided by ChAS) including 270 HapMap samples, as well as 96 healthy normal individuals. The Hidden Markov Model (HMM) available in ChAS was used to determine the copy number state (CN-state) and their breakpoints. The customized high-resolution condition was used as a filter for the determination of CNV: CN-gains with 50 marker count and $400 \mathrm{~Kb}$, and CN-losses with 50 marker count and $100 \mathrm{~Kb}$. We used the median absolute pairwise difference (MAPD) and the single nucleotide polymorphism quality control (SNP-QC) score as the quality control parameters. Only samples with values of MAPD $>0.25$ and SNP-QC $<15$ were included in further analysis.

\section{Bioinformatic analysis}

We developed a Perl script to load the CNV segment data files generated by ChAS for each sample, compare the files to build a table of genes that contains events types (gains or losses), frequencies of altered regions, including chromosomes and cytogenetic bands, and Online Mendelian Inheritance in Man (OMIM) information, and incorporate additional information from different databases: haploinsufficiency information from DECIPHER database of genomic variation, genes reported at dbEMT 2.0, and genes affected in gastric adenocarcinoma from Harmonized Cancer Datasets (Table S1).

The genes altered in at least three patients (cut-off $\geq 3$ ) with DGC, IGC or NAG were included for analysis and visualizations with the language and environment for statistical computing and graphics R v4.0.2 and Bioconductor v3.12 packages. 
The karyotype was created with KaryoploteR and BSgenome.Hsapiens.UCSC.hg19 v1.4.0. The comparison among samples was made using Venn diagrams with the jVenn server and the heatmap with gplots. Enrichment and gene ontology (GO) analysis were performed with the ClusterProfiler v3.16.1 packages, org.Hs.eg.db 3.11.4, enrich plot v1.8.1, and GOplot v1.0.2, with the support of functional enrichment analysis via DAVID v6.8 bioinformatics resources. The profile of altered molecular functions in GC was summarized according to the proportion of CNA-genes and the Molecular Function (MF) gene collection of the gene ontology terms from the DAVID database, adjusted by FDR. We used dot plots, heatmaps, and chord plots to visualize the general GC CNA profiles for DGC, IGC, and NAG.

To identify the main genes and signaling pathways involving CNA EMT-genes, we analyzed and compared GC CNA-genes (cut-off $\geq 3$ ) according to those previously reported in the dbEMT 2.0 accessed on October $12^{\text {th }}, 2020$.

Finally, to establish the profile-associated hallmarks of cancer involving DGC, IGC, and NAG EMT-genes, we generated an interaction network by CNA-type (gains and losses) based on genetic and physical interactions, biological pathways, and predicted relationships using the GeneMANIA prediction server and Cytoscape v.3.8.2, including the manual annotation of their corresponding cancer hallmarks: adhesion, angiogenesis, inflammation, migration, metastasis, morphogenesis, proliferation, and survival (28) with punctual scrutiny and help from databases such as The Human Protein Atlas. For further consultation of the databases, protocols, software, and specific packages used in this study see Table S2.

\section{Results}

\section{Sample characteristics}

Samples from 21 Mexican patients (third-generation Mexicans) between 35 and 91 years old $(61.7 \pm 15.9$ years) without previous cancer treatment (naïve) were included in our study. The data includes 21 samples: seven DGC, seven IGC and seven NAG (control samples). Our data have been deposited in the NCBI Gene Expression Omnibus and assigned the GEO series accession number GSE117093. We have seven adjacent tissue files (.CEL), but these files were not included in data analysis, because some adjacent tissues were contaminated with cancer cells or these were not of the quality required for subsequent analyzes. 
Table 1 shows the identity card (ID) and the percentage of neoplastic cells for tumor tissues ranging between $50-70 \%$. Blood agar culture showed that one IGC and three NAG patients were positive for Helicobacter pylori (data obtained from our biobank database). Tumor size $(\mathrm{T})$, number of lymph nodes $(\mathrm{N})$ and metastasis $(\mathrm{M})$ classification data are also presented in Table 1.

Table 1. Characteristics of GC samples analyzed in this study.

\begin{tabular}{|c|c|c|c|c|c|c|c|}
\hline ID & $\begin{array}{c}\text { Age } \\
\text { (years) }\end{array}$ & Gender & CT & $\% \mathrm{CC}$ & H. pylori & TNM & Treatment \\
\hline 3CG-008 & 72 & M & Intestinal & 70 & Positive & IB T1 N1 M0 & Naïve \\
\hline 3CG-126 & 80 & M & Intestinal & 60 & Negative & IIA T4 N0 M0 & Naïve \\
\hline 3CG-128 & 91 & M & Intestinal & 70 & Negative & IIA T3 N2 M0 & Naïve \\
\hline 3CG-046 & 52 & $\mathrm{~F}$ & Intestinal & 60 & Negative & IV T4 N2 M0 & Naïve \\
\hline 3CG-099 & 59 & M & Intestinal & 50 & Negative & II T3 N0 M0 & Naïve \\
\hline 3CG-146 & 71 & M & Intestinal & 60 & Negative & IIB T3 N2 M0 & Naïve \\
\hline 3CG-104 & 69 & M & Intestinal & 60 & Negative & II A T4 N0 M0 & Naïve \\
\hline 3CG-047 & 58 & M & Diffuse & 70 & Negative & IV T4 N3 M0 & Naïve \\
\hline 3CG-173 & 76 & M & Diffuse & 70 & Negative & II A T2 N3 M0 & Naïve \\
\hline 8CG-004 & 76 & M & Diffuse & 70 & Negative & II T1 N0 M0 & Naïve \\
\hline 1CG-001 & 45 & M & Diffuse & 60 & Negative & IV T4N2M1 & Naïve \\
\hline 3CG-035 & 55 & M & Diffuse & 60 & Negative & IV T4N2M0 & Naïve \\
\hline 3CG-042 & 64 & M & Diffuse & 50 & Negative & IV T4, N2 M0 & Naïve \\
\hline 3CG-064 & 38 & M & Diffuse & 50 & Negative & IV T4 N2 M0 & Naïve \\
\hline 4GB-001 & 64 & M & Gastritis & 0 & Negative & NA & NA \\
\hline
\end{tabular}




$\begin{array}{llllllll}\text { 4GB-031 } & 62 & \text { M } & \text { Gastritis } & 0 & \text { Negative } & \text { NA } & \text { NA } \\ \text { 4GB-015 } & 35 & \text { F } & \text { Gastritis } & 0 & \text { Negative } & \text { NA } & \text { NA } \\ \text { 4GB-025 } & 39 & \text { M } & \text { Gastritis } & 0 & \text { Positive } & \text { NA } & \text { NA } \\ \text { 4GB-033 } & 76 & \text { F } & \text { Gastritis } & 0 & \text { Positive } & \text { NA } & \text { NA } \\ \text { 4GB-036 } & 38 & \text { F } & \text { Gastritis } & 0 & \text { Positive } & \text { NA } & \text { NA } \\ \text { 4GB-042 } & 77 & \text { F } & \text { Gastritis } & 0 & \text { Negative } & \text { NA } & \text { NA }\end{array}$

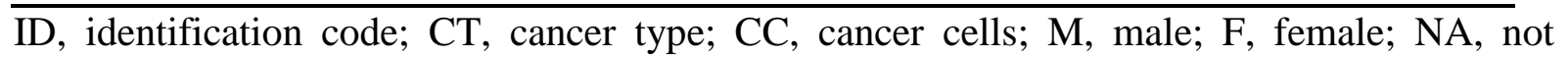
applicable; TNM, T: the extent of the primary tumor; $\mathrm{N}$ : the absence or presence and extent of regional lymph node; M: the absence or presence of distant metastasis (29-31).

\section{Genomic detection of CNA}

We obtained the total number of CNA and classified them as gains or losses for each chromosome in GC and NAG samples. By total CNA, DGC had more CNA than IGC (3505 and 2781, respectively), while there were 828 events in NAG samples. By tissue, we observed more gains than losses (G/L) in both cancer types, DGC (2310/1195) and IGC (1550/1231), but the opposite occurred in NAG (375/453) (Table S3).

To identify the most relevant CNA in GC and NAG, we analyzed alterations occurring in at least three patients (cut-off $\geq 3$ ). This comparison showed a similar pattern for total CNA with more events in DGC (710), IGC (590), and NAG (332). Also, we observed more gains than losses (G/L) in DGC (516/194), IGC (314/276) and even in NAG (196/136), unlike when all patients were included. In addition, DGC had the highest number of gains and IGC had the highest number of losses (Table S3). In Table 2 we show chromosomes and sizes with gain and loss numbers, representative and summarized.

Table 2. Principal affected chromosomes by CNA cumulative length in diffuse gastric cancer, intestinal gastric cancer and non-atrophic gastritis. 


\begin{tabular}{|c|c|c|c|c|}
\hline Type & Chromosome & Gains & Losses & $\begin{array}{l}\text { Length } \\
\text { (Mb-cl) }\end{array}$ \\
\hline \multirow[t]{3}{*}{ DGC } & 1 & 327 & ----- & 117.9 \\
\hline & 4 & ----- & 155 & 40.8 \\
\hline & 5 & ----- & 148 & 74.23 \\
\hline \multirow[t]{3}{*}{ IGC } & 1 & ----- & 148 & 33.78 \\
\hline & 8 & 365 & ----- & 139.8 \\
\hline & $X$ & ----- & 66 & 167.1 \\
\hline \multirow[t]{6}{*}{ NAG } & 6 & ----- & 28 & 0.40 \\
\hline & 7 & 21 & ----- & 0.20 \\
\hline & 14 & 10 & ----- & 3.02 \\
\hline & 17 & ----- & 15 & 1.86 \\
\hline & $X$ & ----- & 87 & 0.47 \\
\hline & $\mathrm{X}$ & 207 & ----- & 1.20 \\
\hline
\end{tabular}

DGC: Diffuse gastric cancer; IGC: Intestinal gastric cancer; NAG: Non-atrophic gastritis; Mbcl: Megabases cumulative length.

To visualize the distribution of DGC and IGC chromosome gains and losses, we plotted the identified CNA present in a karyogram (cut-off $\geq 3$ ), locating alterations according to the coordinates of the Human genome hg19 (Figure 1). The top five altered cytobands are shown in Tables 3 and S4. 
Table 3. Top five altered cytobands in diffuse gastric cancer, intestinal gastric cancer and non-atrophic gastritis.

\begin{tabular}{|c|c|c|c|c|}
\hline Type & Cytoband & Gains & $\begin{array}{l}\text { Length } \\
\text { (Mb-cl) }\end{array}$ & Number of patients \\
\hline \multirow[t]{5}{*}{ DGC } & $\mathrm{Xq} 28$ & 37 & 11.5 & 6 \\
\hline & $8 q 24.22$ & 25 & 15.8 & 5 \\
\hline & 1q32.1 & 26 & 12.16 & 5 \\
\hline & $8 \mathrm{q} 24.3$ & 25 & 17.46 & 6 \\
\hline & $1 \mathrm{q} 23.3$ & 22 & 15.26 & 6 \\
\hline \multirow[t]{5}{*}{ IGC } & $8 q 24.3$ & 24 & 12.662 & 4 \\
\hline & $13 q 34$ & 18 & 4.9018 & 4 \\
\hline & $8 \mathrm{q} 24.21$ & 18 & 6.2619 & 4 \\
\hline & $8 \mathrm{q} 24.22$ & 16 & 6.4856 & 4 \\
\hline & $8 q 12.1$ & 16 & 5.8523 & 4 \\
\hline \multirow[t]{5}{*}{ NAG } & $\mathrm{Xq} 26.2$ & 24 & 34.026 & 6 \\
\hline & $\mathrm{Xq} 21.2$ & 22 & 33.586 & 7 \\
\hline & $\mathrm{Xp} 22.33$ & 19 & 167.504 & 7 \\
\hline & $\mathrm{Xq} 23$ & 16 & 63.79 & 6 \\
\hline & $\mathrm{Xq} 26.3$ & 13 & 217.774 & 7 \\
\hline
\end{tabular}


DGC: Diffuse gastric cancer; IGC: Intestinal gastric cancer; NAG: Non atrophic gastritis; Mbcl: Megabases cumulative length.

Interestingly, in DGC and IGC the most frequent CNA lengths were 100-200 Kb, while 1-50 $\mathrm{Kb}$ were more common in NAG, considering gains and losses (Table S5).

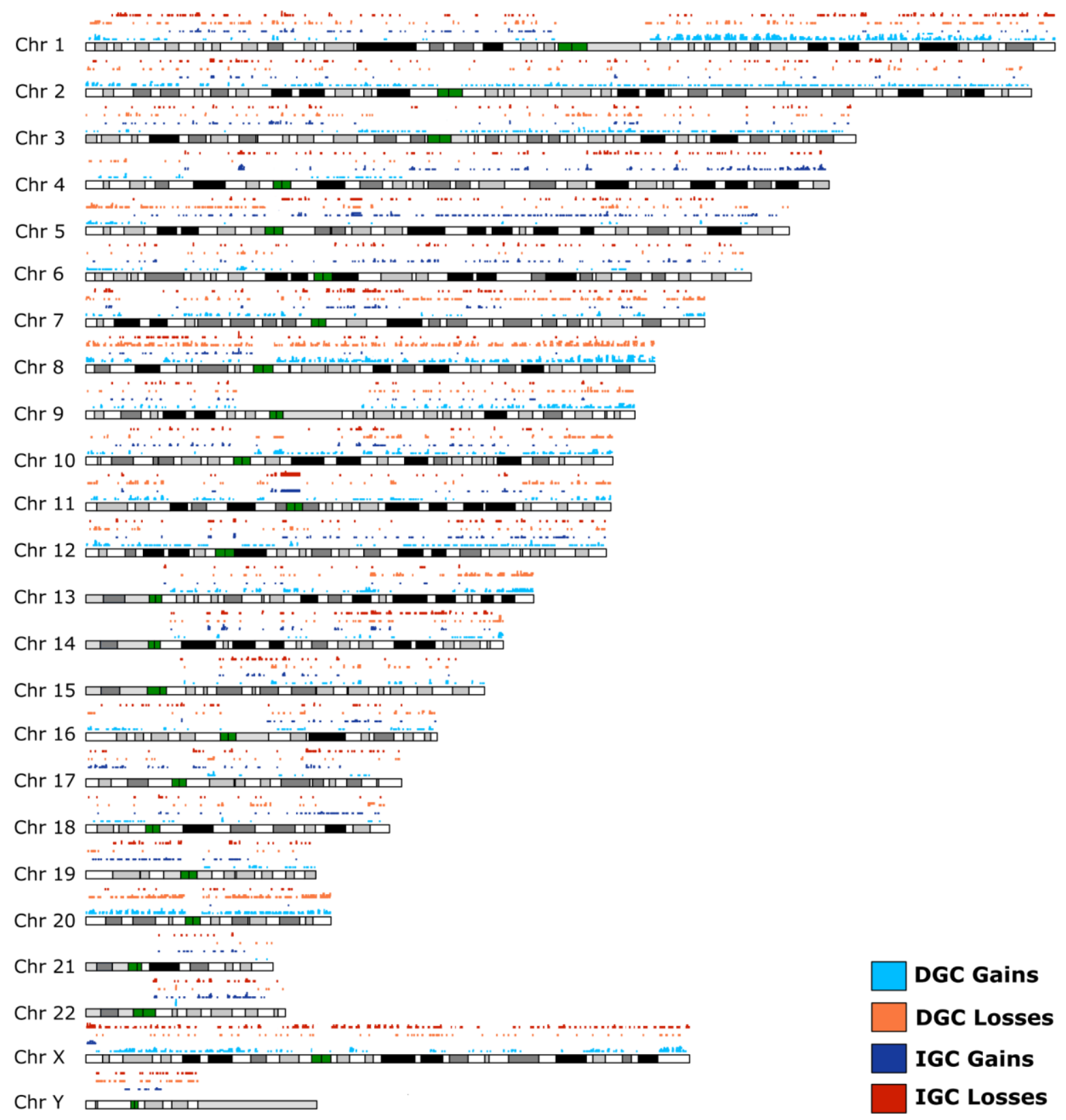

Figure 1. Karyogram with CNA distribution in diffuse and intestinal gastric cancers. CNA

events (gains or losses) were present from chromosomes 1 to $22, \mathrm{X}$, and Y. Gains (blue and 
dark blue) and losses (orange and red) are plotted for $\geq 3$ DGC or IGC patient samples. Cytobands (gray, black or white bars) and centromeres (green bars) are shown.

\section{Gastric cancer genes associated with $\mathrm{CNA}$}

Overall, we found 2441 CNA-genes in DGC-IGC-NAG. GC had 2420 affected genes (99\%) while only 108 genes (4\%) were affected in NAG; some of these alterations were shared between GC and NAG. We observed 1317 unique CNA-genes in DGC, 596 in IGC, and 21 in NAG; both cancer types shared 420 genes, while 60 genes were shared between GC and NAG. In addition, 19 NAG genes were shared with DGC and eight genes with IGC (Figure 2A and Table S6).

To identify possible emerging patterns among samples, we performed hierarchical clustering heatmaps (Figure 2B). Results showed the molecular signature and hierarchical clustering of samples according to 2441 genes. The emerging pattern of altered genes affected by CNA distinguishes DGC and IGC from NAG.
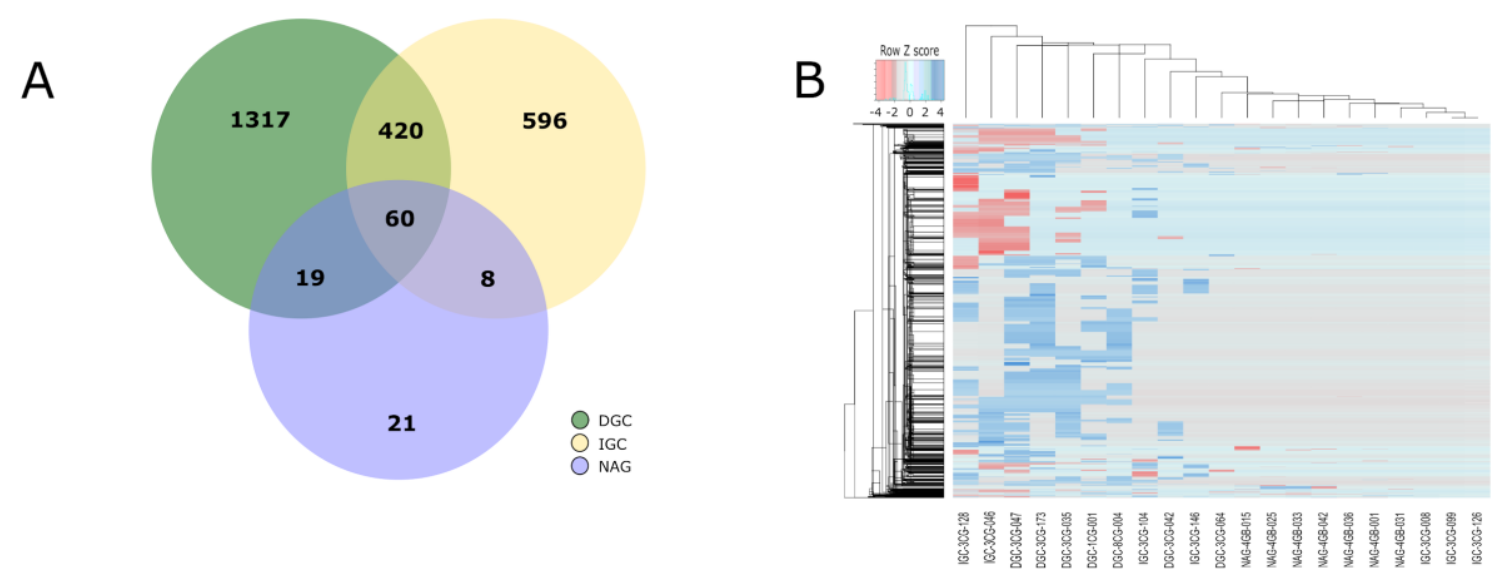

Figure 2. Profile of CNA-genes in gastric cancer from $\geq 3$ patients. (A) The Venn diagram presents frequencies of specific and shared genes in diffuse gastric cancer (DGC), intestinal gastric cancer (IGC) or non-atrophic gastritis (NAG) in CNA. (B) The heatmap shows the hierarchical clustering of gains (blue), losses (red), and no alterations (light blue and light red).

\section{GO analysis of gastric cancer}


We obtained the functional profile for GC through enrichment and gene ontology (GO) analysis of 1317 genes altered only in DGC and 596 in IGC. To identify the principal molecular functions altered in GC, we categorized these CNA-genes, independently of GC-type, in two groups: gains and losses (Figure 3A and 3B). The top ten molecular functions (MF) associated with CNA gains or losses revealed that transcription activator, tyrosine kinase activity, growth factors and hormone binding, as well as intracellular signal transduction genes were enriched in GC. Gene-losses mainly involved transcription coactivator and serine/threonine kinase activity, as well as, several receptors binding to hormone, steroid hormone, nuclear receptor, beta-catenin, intermediate filament, mitogen-activated protein kinase binding genes. In addition, we identified the principal CNA-genes affecting the MF by GC type: DGC and IGC (Figure 3C, 3D, and 3E). 


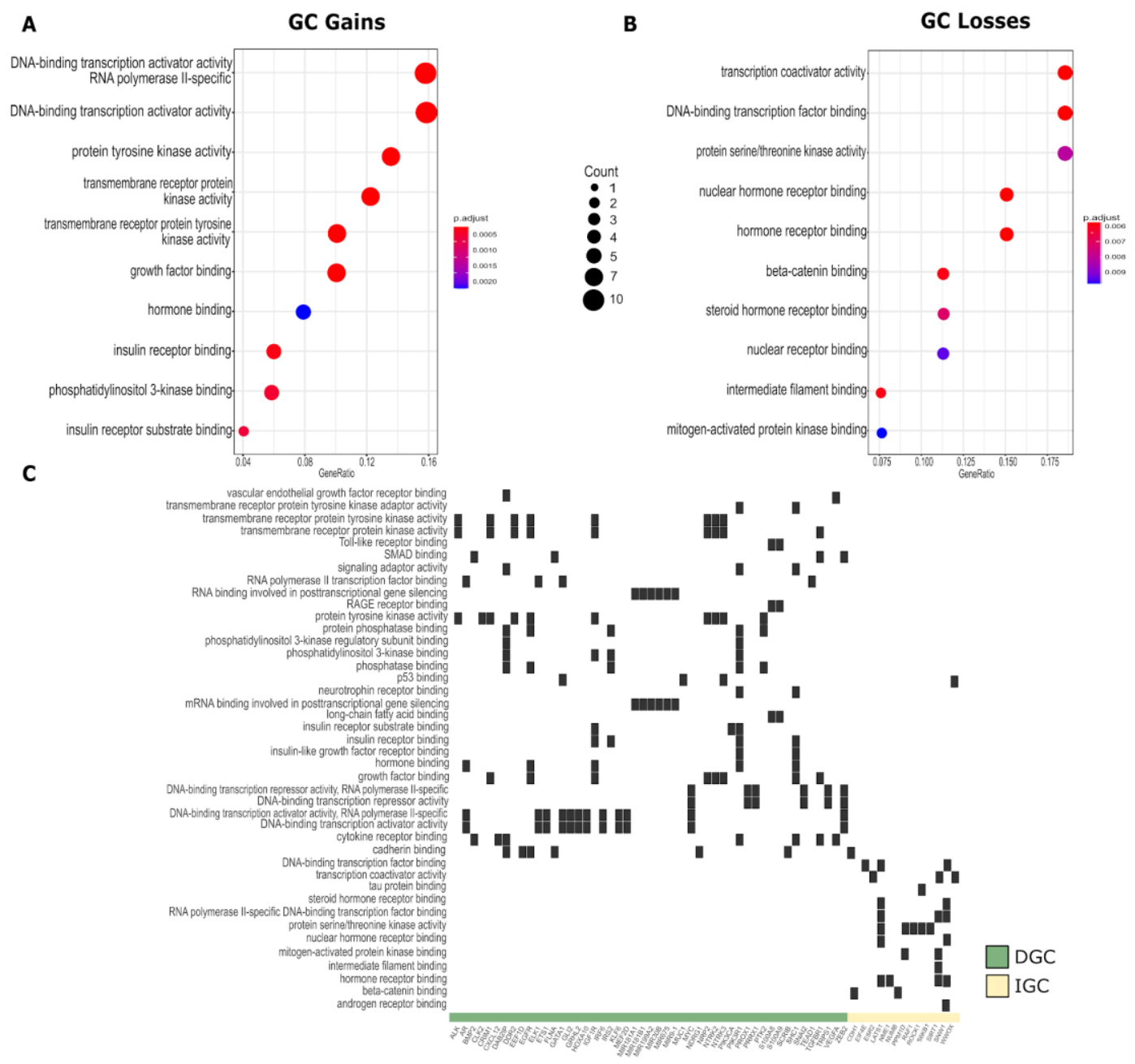

D

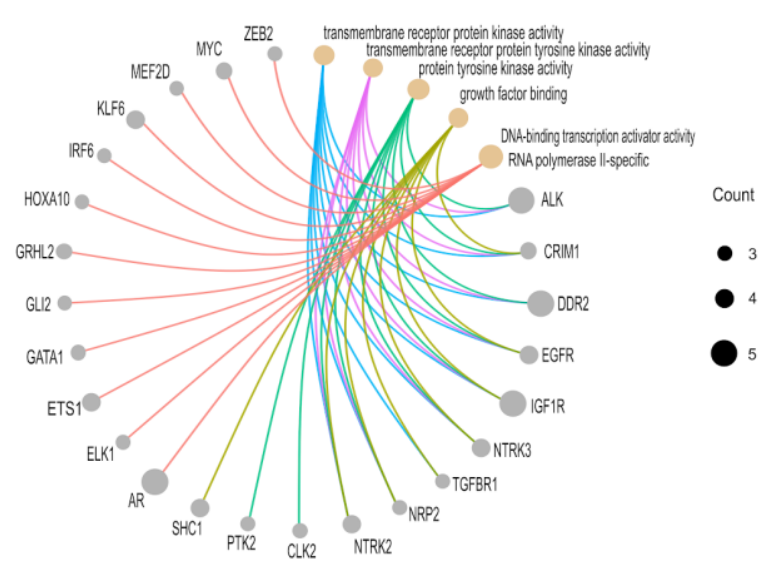

E

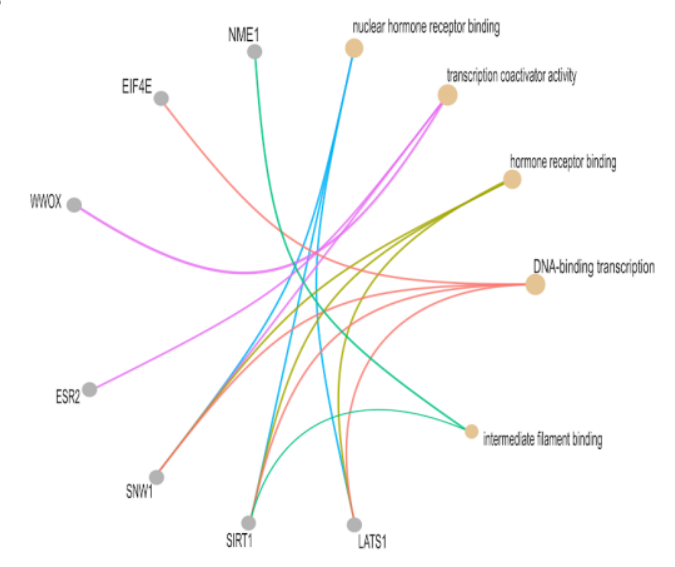

Figure 3. Molecular function profiles of gastric cancer CNA-genes. Gene enrichment analysis affected by CNA-genes in $\geq 3$ gastric cancer (GC) patients. It is summarized by CNAtype with dot plots for gains (A) and losses (B), according to the proportion of CNA-genes and Molecular Functions (MF) gene collection of the gene ontology (GO) terms from the DAVID 
database, adjusted by FDR. The heatmap (C) shows the main MF altered by CNA-genes in Diffuse-GC and Intestinal-GC. The CNA-genes-MF networks depict the possible relationship between CNA gains (D) and losses (E) within GC. Gene-ratio (M/N) is the proportion between CNA genes in $\geq 3 \mathrm{GC}$ patients $(\mathrm{M})$ and the collection of genes from the GO term database function (N). Count (circle sizes) represents the number of CNA-genes associated with MF.

\section{CNA-EMT genes in DGC and IGC}

To identify the main genes and signaling pathways involving CNA-EMT genes in GC and NAG, we compared GC CNA-genes against a comprehensive and annotated database of epithelial-mesenchymal transition genes (dbEMT). We found 551 CNA-EMT genes for DGC, 619 for IGC and 28 for NAG. With a cut off $\geq 3$ in DGC-112, IGC-66 and NAG-5. The complete data of EMT-genes for DGC, IGC, and NAG with chromosome and cytoband locations, CNA-type (gain or loss), and $p$-values can be found in Table S1.

\section{GO Analysis of EMT-genes}

We performed a gene ontology (GO) enrichment analysis to determine the MF of the main CNA-EMT genes affected in DGC, IGC, and NAG (Figure 4). Our analysis shows that CNAEMT gene-gains in DGC involved transmembrane receptor tyrosine kinase, DNA and RNA binding, several receptor binding: insulin, growth factor, toll-like, hormone, as well as SMAD, p53, chromatin, calcium ion binding and microtubule binding (Figure 4A). CNA-EMT genelosses included DNA and chromatin binding, nuclear hormone receptor binding, beta-catenin, steroid hormone, mitogen-activated protein binding, intermediate filament binding, p53 binding, RNA polymerase II-specific DNA binding (Figure 4B). Moreover, CNA-EMT genegains in IGC involved insulin receptor substrate and phosphatase binding, kinase regulation, neurotrophin receptor binding, 1-phosphatidylinositol-3-kinase activity, transmembrane receptor protein tyrosine kinase adaptor activity, and VEGF receptor binding; while CNA-EMT gene-losses in IGC involved only coenzyme binding and transcription coactivator activity (Figure 4C). On the other hand, the main MF for CNA-EMT gene-gains in NAG included transcription regulatory region DNA, transcriptional activator activity RNA, armadillo repeat and $\mathrm{C} 2 \mathrm{H} 2$ zinc finger domain binding, gamma- and beta- catenin binding, as well as cysteinetype endopeptidase inhibitor activity involved in apoptotic process and estrogen receptor and 
bioRxiv preprint doi: https://doi.org/10.1101/2021.11.22.469612; this version posted November 22, 2021. The copyright holder for this preprint (which was not certified by peer review) is the author/funder, who has granted bioRxiv a license to display the preprint in perpetuity. It is made available under aCC-BY-NC-ND 4.0 International license.

steroid hormone receptor activity; while CNA-EMT gene-losses in NAG involved damaged DNA, WW domain, and p53 binding as well as DNA-binding transcription activator activity (Figure 4D). 
bioRxiv preprint doi: $\mathrm{https}$ //doi.org/10.1101/2021.11.22.469612; this version posted November 22, 2021. The copyright holder for this preprint (which was not certified by peer review) is the author/funder, who has granted bioRxiv a license to display the preprint in perpetuity. It is made available under aCC-BY-NC-ND 4.0 International license.
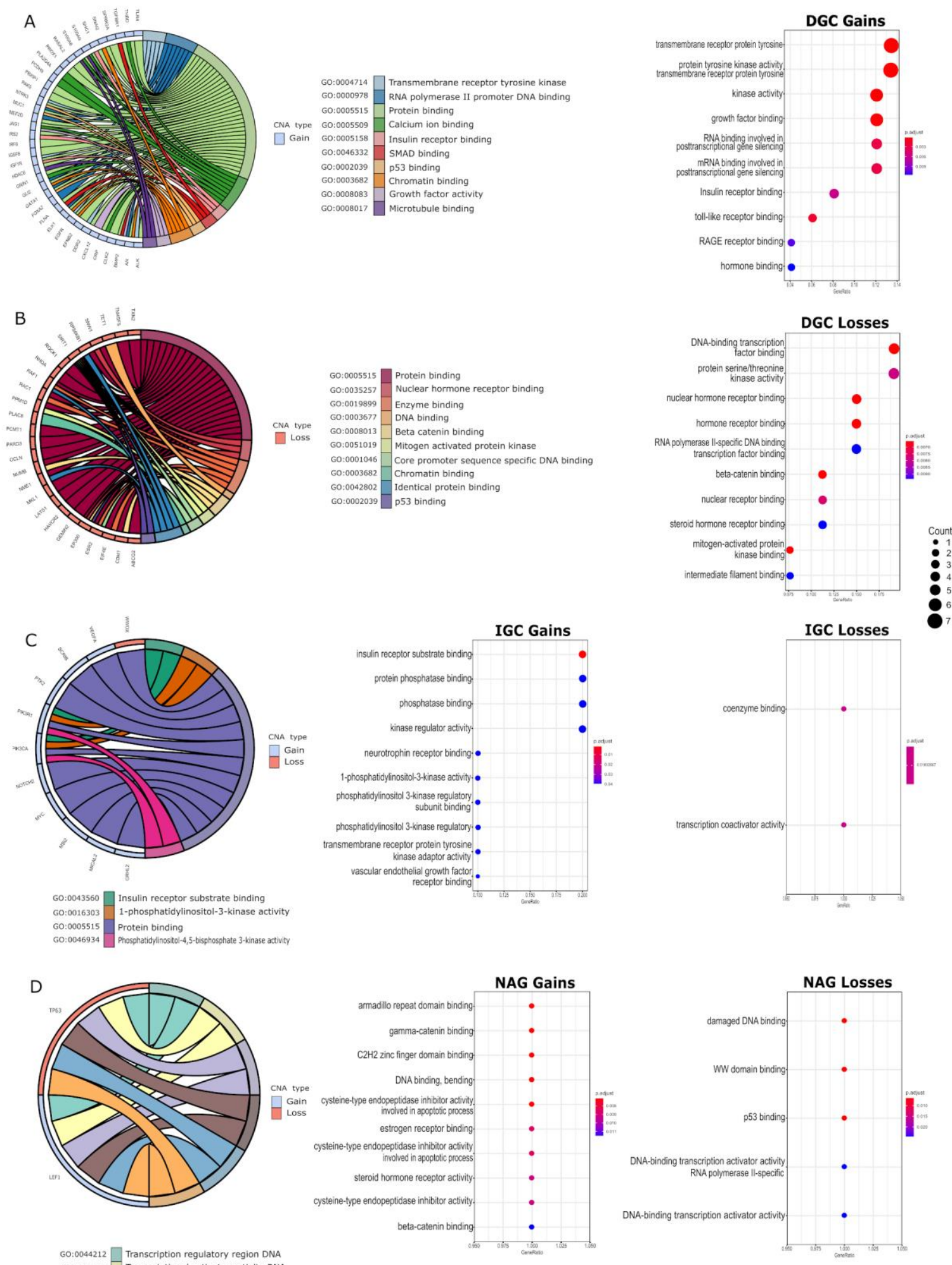

\begin{tabular}{l|l} 
60:0094212 & Transcription regulatory region DNA \\
60:0001077 & Transcriptional activator activity RNA \\
60:0003682 & Chromatin binding \\
\hline 60:0043565 & Sequence-specific DNA \\
\hline 60:0003700 & Transcription factor activity \\
60:0003677 & DNA binding binding
\end{tabular} 
Figure 4. Principal molecular functions associated with CNA-EMT genes in gastric cancer and non-atrophic gastritis. The main MF associated with CNA-EMT are represented with chord plots and dot graphics for (A) DGC gains, (B) DGC losses, (C) IGC gains or losses, and (D) NAG gains or losses. Chord plots (left panels) show associations between genes and molecular functions, indicating their CNA-type by color coding (gains/blue and losses/red). Dot plots (right panels) show an enrichment analysis of molecular functions, and loss or gain genes counts in samples. CNA, copy number alteration; EMT, epithelial mesenchymal transition; DGC, diffuse gastric cancer; IGC, intestinal gastric cancer; NAG, non-atrophic gastritis.

\section{CNA-EMT genes associated hallmarks of cancer}

Based on the main molecular profile of altered CNA-EMT genes in GC and NAG, we generated the functional network between 39 previously selected unique CNA-EMT genes, (19 genes for DGC, seven for IGC, 11 common to GC, and two for NAG; cut-off $\geq 3$ patients). Gained genes with the highest degree, at least four interactions per gene, were EGFR, MICAL2, MYC, NDRG1, and PIK3R1, while lost genes included GLI2, EP300 and PTPN11. The principal functions related to these CNA-EMT genes have been previously associated with several hallmarks of cancer: adhesion, angiogenesis, inflammation, migration, metastasis, morphogenesis, proliferation, and survival (Figure 5). 


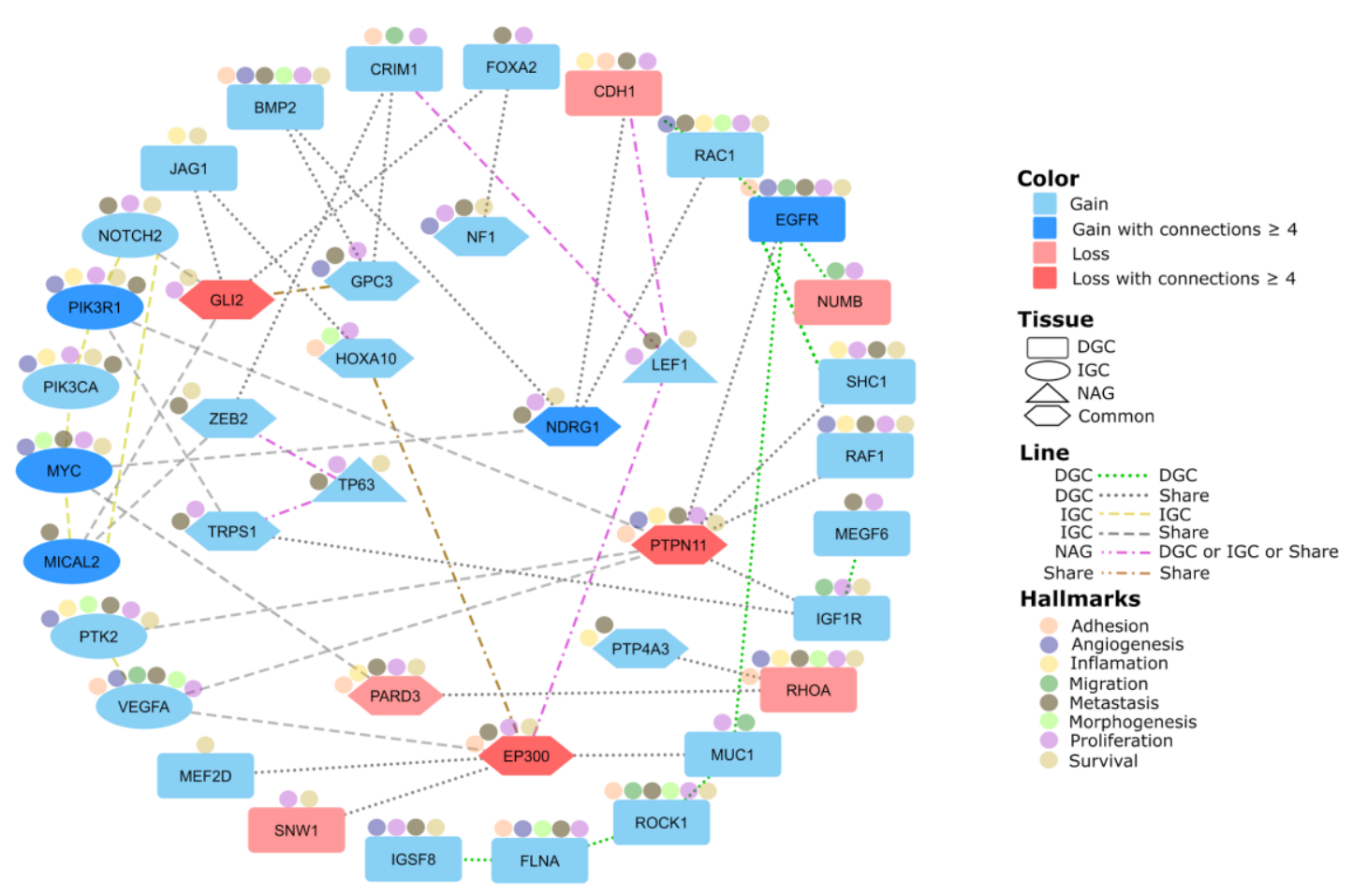

Figure 5. Gastric cancer and non-atrophic gastritis CNA-EMT genes network and associated hallmarks of cancer. The functional interactions among CNA-EMT genes identified in DGC (rectangles), IGC (ovals), and shared genes (hexagons) are identified by their CNA-type (gains/blue and losses/red) and associated hallmarks of cancer (colored dots). DGC, diffuse gastric cancer; IGC, intestinal gastric cancer; NAG, non-atrophic gastritis; EMT, epithelial-mesenchymal transition; CNA, copy number alterations.

\section{Discussion}

This is the first whole-genome high-density array study in GC from Mexican patients in three groups: DGC, IGC, and NAG as non-cancerous control. Using this experimental strategy, it was possible to generate a karyogram and obtain molecular signatures for diffuse and intestinal GC, and their relationship with CNA-EMT genes independently of age, gender, \% CC, H. pylori presence/absence, TNM, and treatment (naïve samples in our set). In addition, our genomic analysis was focused on the molecular profile of GC, particularly involving alterations of EMTgenes, given their role in cancer progression as epithelial cell transformation to mesenchymal cells is fundamental to metastasis (32) and chemoresistance $(33,34)$. Our results coincide with those previously reported in the literature, which provides validity and robustness to our 
findings and allows us to report novel data or not yet explored potential diagnostic, prognostic, and treatment response markers.

Globally, the alteration profile in GC was dominated by gains. This phenomenon, where gains are more abundant than losses, has been previously reported in different tumor lines, including gastric cell lines (35). Chromosomal gains in cancer might result in increased gene functions, providing cancer cells a competitive advantage for the development of metastasis (36), while chromosomal losses might involve the down-regulation of tumor suppressor genes (37), disrupting homeostasis and accelerating cancer progression. The most affected CNAchromosomes for DGC were 1, 4, and 5; for IGC 1, 8, and X, and for NAG 6, 7, 14, 17, and X. The altered cytobands associated with GC found in this work (Table 3) are in agreement with previous studies; as an example, $8 \mathrm{q} 24$ has been implicated in the development of different tumors (38). The highest frequencies of gains in advanced GC were found at 8q24.21 (65\%) and 8q24.3 (60\%), and the pattern of CNA in advanced GC was quite different from that in early GC, this increased CNA numbers is associated with disease progression from early to advanced GC (39). The 8q24 cytoband has also been reported in Latin American countries such as Brazil (40) and Venezuela (41), as well as in Asian countries like Korea (42).

Interestingly, the most frequent CNA length in GC was 100-200 Kb in both DGC and IGC versus $1-50 \mathrm{~Kb}$ in NAG. The biological implications of this alteration length pattern in GC compared to non-cancerous tissues such as NAG is yet to be determined. Meanwhile, it is important to highlight that a resolution of $100-200 \mathrm{~Kb}$ versus $\mathrm{Mb}$ is an advantage of molecular resolution approaches over classical cytogenetics (CGH, FISH, among others) to discover "small" potentially important alterations in cancer samples.

The cumulative length averages $(\mathrm{Mb}-\mathrm{cl})$ of these alterations were 183.44 for DGC, 113.56 for IGC, and 1.19 for NAG. These lengths, whether gained or lost, describe the magnitude of global alterations per tissue; yet, their relevance lies on the molecular functions, biological process, and interaction networks in which they participate.

We identified a molecular profile that distinguishes GC from NAG, based on 2441 genes affected by CNA. They are associated with GC, as well as the differences and similarities between histological subtypes, DGC (undifferentiated) and IGC (well-differentiated) compared to a non-cancerous tissue such as NAG (43). Interestingly, we identified 60 affected genes shared between GC and NAG, 19 were shared exclusively with DGC while only eight with 
IGC. This emerging pattern of shared altered genes between cancerous and non-cancerous tissues, should be further studied to identify possible CNA-dependent oncogenic pathways and progression trajectories from NAG to either GC subtype, particularly in conjunction with environmental factors such as $H$. pylori infection, diet, and lifestyle, that might be involved in spread patterns affecting patient survival (43).

In the heatmap (Figure 3B) a separation between NAG and GC is observed, showing clusters based on the molecular profiles of CNA-genes. There is a greater heterogeneity between the IGC samples in clusters, but there are more genes affected in DGC. The front-line tool for IGC distinction has been based on different criteria such as the histopathological classification proposed by Lauren. However, due to the challenges, conflicts in the correct assignment, diagnosis and treatment new criteria have been proposed such as the molecular characterization by The Cancer Genome Atlas Research Network (TCGA), which divides GC into four subtypes (44). Our observations agree with the need for new proposals for the classification of GC, which includes defined subgroups with the integration of several genomic and genetic parameters where CNA are present.

We analyzed and determined the molecular function profile of GC CNA-genes. Concerning gains, we observed increased alterations involving transcription, signaling, tyrosine kinases, growth factors, hormones, insulin; while in losses, molecules involved in transcription, serine/threonine and MAP kinases, hormones, steroids, beta-catenin binding, filament binding were decreased. These gene sets are important in GC biology. CNA-IGC genes were 13, e.g. CDH1, LAST1, ROCK1, and WWOX; and CNA-DGC genes were 49, e.g. CRIM1, EGFR, MIR9-1, MUC1, MYC, NDRG1, SCRIB, SNAI2, VEGF, and ZEB2, hence, we focus on these example genes with the intention of comparing our findings with others and placing them in a biologically coherent context. For example, $\mathrm{CDH} 1$ codes for E-cadherin and, from a simplified viewpoint, E-cadherin maintains the epithelial phenotype; if $\mathrm{CDH} 1$ is lost, this promotes the mesenchymal phenotype, i.e., it favors loss of adhesion and metastasis (32).

We performed an enrichment analysis of unique CNA-genes for all tissues and observed several shared molecular functions, such as protein binding. Some gained genes coding for RNAbinding proteins (RBP) (45) have diverse targets and participate in tumor progression by regulating homeostasis and changing expression patterns. Chromatin binding is another altered function in $\mathrm{GC}$ that participates in regulating eukaryotic gene expression, methylation profiles 
modulation, and genome stability maintenance (46). EMT is a process that involves changes in histone modification, DNA methylation, and chromatin accessibility. These changes can be promoted through transcription, allowing the cell to have an identity or to have a MET-EMT conversion (47). The kinase function in DGC and IGC gains (48) have recently been considered key regulators in the development of cancer. Many kinases are related to the initiation and progression of carcinogenesis, and are one of the main therapeutic targets for the development of inhibitors in the clinical area. Kinases are able to promote EMT and enhance invasion, migration, and evasion of apoptosis (49). In IGC, we highlight PIK3R1 and PIK3CA. The PI3K pathway is a key regulatory hub for cell growth, survival and metabolism (50). Activation of $\mathrm{PI} 3 \mathrm{~K}$ is a frequent hallmark of cancer, highlighted by the prevalence of somatic mutations in genes encoding key components of this pathway (51). These enzymes are responsible for transferring a phosphate group; however, the reverse process is carried out by phosphatases which also are particularly affected in IGC. PIK3R1, is a gene frequently affected by mutations or copy numbers in various types of cancer according to the TCGA project. These genes converge on the PI3K/AKT/mTOR pathway, involved in the regulation of many processes (51). At present, the differences between DGC and IGC have been insufficiently explored and understood; differences in etiology, location, incidence, genetic profiles, among others, have been observed (52). The GC CNA-EMT network (Figure 5) was generated with relevant genes according to different criteria: frequency among patients as well as genetic connections, reported pathways, and experimental associations with several databases: EMTDB, The Human Protein Atlas, COSMIC (53), Cancer Hallmark Genes (CHG) database (54). Shared and exclusive altered genes were observed for each tissue-type. The common CNA-EMT genes between DGC and IGC include GLI2, associated with proliferation (55); EP300, with multiple functions as an inhibitor of anti-tumor immune response via metabolic modulation (56); PTPN11, associated with GC progression; and NDRG1, associated with metastasis and poor prognosis in GC (57). A relevant gene in DGC is EGFR, which given its consistent CNV-GC association, is now the target for the development of anti-GC therapies (58). IGC-exclusive EMT-genes are MICAL2, MYC, and PIK3R1. MICAL2, a destabilizing F-actin in cytoskeletal dynamics, has been found in poor prognosis GC (59). MYC gains have also been reported in several GC studies, as expected for a common oncogenic gene (60) associated with proliferation, differentiation, and apoptosis (61). PIK3R1 participates in the PI3K/AKT 
signaling pathway with roles in apoptosis and cell survival, as well as chemotherapy resistance in GC (62).

A large amount of data remains to be analyzed, including $\mathrm{LOH}$, mosaicism, and other gene sets that participate in different hallmarks of cancer. Another limitation of our study was the absence of the transcriptomic exploration to validate our GC EMT-signature, particularly for DGC and IGC. Yet, the concordance of CNA with expression alterations of EMT-related genes is plausible, as previously observed for multiple cancer types from The Cancer Genome Atlas (TCGA) data (35). Also, further inclusion of precancerous stages would allow us to depict the "profile" of IGC progression. The results from our genomic approach, coincide with those already reported in the literature, which gives validity and solidity to our results. After all, this strategy allowed us to report novel data, scarcely or not yet explored, to identify differential GC CNA, associate them to relevant molecular functions related to the hallmarks of cancer, and predict the EMT-signature for DGC and IGC. We believe that targeting these networks will potentially serve as diagnostic, and prognostic markers. Additionally, the use of NAG as a nonmalignant control allowed us to study the molecular and cellular events of GC, and identify possible biomarkers for the "early" gastric cancer stages.

\section{Acknowledgments}

The authors are grateful to Irma P. Ramos-Vega, Brian-Alexander Cruz-Ramírez, and Alejandra García-Bejarano for technical assistances.

\section{Availability of data and materials}

All data generated or analyzed during this study are included in this published article. The data have been deposited in the Gene Expression Omnibus (GEO; https://www.ncbi.nlm.nih.gov/geo/) using accession number: GSE117093.

\section{Authors' contributions}

VLS, HAVS, and JDME: Carried out molecular techniques, participated in the analysis of results, preparation, writing, and discussion of the manuscript. JT, MCP, and PPS: Responsible for the clinical aspects of the study, including the recruitment of patients; participated in data discussion and edition of the manuscript. MERT: Responsible for the design of the study. 
Supervision of the experimental work. Critical review, editing, and writing of the manuscript. JT and MERT responsible for acquiring financial support. All authors read and approved the final manuscript and agreed to be accountable for all aspects of the work in ensuring that questions related to the accuracy or integrity of any part of the work are appropriately investigated and resolved.

\section{References}

1. Sung H, Ferlay J, Siegel RL, Laversanne M, Soerjomataram I, Jemal A, et al. Global Cancer Statistics 2020: GLOBOCAN Estimates of Incidence and Mortality Worldwide for 36 Cancers in 185 Countries. CA Cancer J Clin. 2021;71:209-49.

2. INEGI - Comunicación Social. Estadísticas a propósito del...día mundial contra el cáncer (4 de febrero) - Datos Nacionales [Internet]. INEGI; 2018 Feb. Report No.: 61/18. Available from: https://www.inegi.org.mx/contenidos/saladeprensa/aproposito/2018/cancer2018_Nal.pdf

3. Laurén P. The two histological main types of gastric carcinoma: diffuse and so-called intestinal-type carcinoma: An attempt at a histo-clinical classification. Acta Pathol Microbiol Scand. 1965;64:31-49.

4. Espejo Romero H, Navarrete Siancas J. [Classification of stomach adenocarcinomas]. Rev Gastroenterol Peru. 2003;23:199-212.

5. Piazuelo MB, Epplein M, Correa P. Gastric Cancer: An Infectious Disease. Infect Dis Clin North Am. 2010;24:853-69.

6. Nagini S. Carcinoma of the stomach: A review of epidemiology, pathogenesis, molecular genetics and chemoprevention. WJGO. 2012;4:156-69.

7. McLean MH, El-Omar EM. Genetics of gastric cancer. Nat Rev Gastroenterol Hepatol. 2014;11:664-74.

8. Mikhail FM, Biegel JA, Cooley LD, Dubuc AM, Hirsch B, Horner VL, et al. Technical laboratory standards for interpretation and reporting of acquired copy-number abnormalities and copy-neutral loss of heterozygosity in neoplastic disorders: a joint consensus recommendation from the American College of Medical Genetics and Genomics (ACMG) and the Cancer Genomics Consortium (CGC). Genet Med. 2019;21:1903-16.

9. Wallander K, Eisfeldt J, Lindblad M, Nilsson D, Billiau K, Foroughi H, et al. Cell-free tumour DNA analysis detects copy number alterations in gastro-oesophageal cancer patients. PLoS One. Public Library of Science (PLoS); 2021;16:e0245488. 
10. Han B, Ren D, Mao B, Song X, Yang W, Zhang H, et al. Tumor copy number alteration (CNA) burden as a prognostic factor for overall survival in Chinese gastric cancers. $\mathrm{J}$ Clin Oncol. American Society of Clinical Oncology (ASCO); 2019;37:e15555.

11. Milne AN. Early-onset gastric cancer: Learning lessons from the young. WJGO. 2010;2:59.

12. Iafrate AJ, Feuk L, Rivera MN, Listewnik ML, Donahoe PK, Qi Y, et al. Detection of large-scale variation in the human genome. Nat Genet. 2004;36:949-51.

13. Seabra AD, Araújo TMT, Mello FAR, Di Felipe Ávila Alcântara D, de Barros AP, de Assumpção PP, et al. High-density Array Comparative Genomic Hybridization Detects Novel Copy Number Alterations in Gastric Adenocarcinoma. Anticancer Res. International Institute of Anticancer Research; 2014;34:6405-15.

14. Morales-Guerrero SE, Rivas-Ortiz CI, Ponce de León-Rosales S, Gamboa-Domínguez A, Rangel-Escareño C, Uscanga-Domínguez LF, et al. Translation of gastric disease progression at gene level expression. J Cancer. 2020;11:520-32.

15. Nadauld LD, Garcia S, Natsoulis G, Bell JM, Miotke L, Hopmans ES, et al. Metastatic tumor evolution and organoid modeling implicate TGFBR2 as a cancer driver in diffuse gastric cancer. Genome Biol. BioMed Central; 2014;15:1-18.

16. Vasaikar SV, Deshmukh AP, den Hollander P, Addanki S, Kuburich NA, Kudaravalli S, et al. EMTome: a resource for pan-cancer analysis of epithelial-mesenchymal transition genes and signatures. Br J Cancer. Nature Publishing Group; 2020;124:259-69.

17. Varga J, Greten FR. Cell plasticity in epithelial homeostasis and tumorigenesis. Nat Cell Biol. 2017;19:1133-41.

18. Kalluri R, Weinberg RA. The basics of epithelial-mesenchymal transition. J Clin Invest. 2009;119:1420-8.

19. Christofori G. New signals from the invasive front. Nature. 2006;441:444-50.

20. Murai T, Yamada S, Fuchs BC, Fujii T, Nakayama G, Sugimoto H, et al. Epithelial-tomesenchymal transition predicts prognosis in clinical gastric cancer: EMT in clinical gastric cancer. J Surg Oncol. 2014;109:684-9.

21. Xia P, Xu X-Y. Epithelial-mesenchymal transition and gastric cancer stem cell. Tumour Biol. 2017;39:101042831769837.

22. Wang G, Anastassiou D. Pan-cancer driver copy number alterations identified by joint expression/CNA data analysis. Sci Rep. 2020;10:17199.

23. Owen GI, Pinto MP, Retamal IN, Fernádez MF, Cisternas B, Mondaca S, et al. Chilean Gastric Cancer Task Force: A study protocol to obtain a clinical and molecular classification of a cohort of gastric cancer patients. Medicine . 2018;97:e0419. 
24. Araújo TM, Seabra AD, Lima EM, Assumpção PP, Montenegro RC, Demachki S, et al. Recurrent amplification of RTEL1 and ABCA13 and its synergistic effect associated with clinicopathological data of gastric adenocarcinoma. Mol Cytogenet. 2016;9:52.

25. Eltoum I, Fredenburgh J, Myers RB, Grizzle WE. Introduction to the Theory and Practice of Fixation of Tissues. J Histotechnol. 2001;24:173-90.

26. Fischer I, Cunliffe C, Bollo RJ, Weiner HL, Devinsky O, Ruiz-Tachiquin M-E, et al. Glioma-like proliferation within tissues excised as tubers in patients with tuberous sclerosis complex. Acta Neuropathol. 2008;116:67-77.

27. Utrera-Barillas D, Valdez-Salazar H-A, Gómez-Rangel D, Alvarado-Cabrero I, Aguilera $\mathrm{P}$, Gómez-Delgado A, et al. Is human cytomegalovirus associated with breast cancer progression? Infect Agent Cancer. 2013;8:12.

28. Hanahan D, Weinberg RA. Hallmarks of Cancer: The Next Generation. Cell. 2011;144:646-74.

29. Jeon J, Cheong J-H. Clinical Implementation of Precision Medicine in Gastric Cancer. J Gastric Cancer. 2019;19:235-53.

30. Zubarayev M, Min E-K, Son T. Clinical and molecular prognostic markers of survival after surgery for gastric cancer: tumor-node-metastasis staging system and beyond. Transl Gastroenterol Hepatol. 2019;4:59-59.

31. Brierley JD, Gospodarowicz MK, Wittekind C. TNM Classification of Malignant Tumours: Edition 8. John Wiley \& Sons; 2017.

32. Bure IV, Nemtsova MV, Zaletaev DV. Roles of E-cadherin and Noncoding RNAs in the Epithelial-mesenchymal Transition and Progression in Gastric Cancer. Int J Mol Sci. 2019;20:2870.

33. Marin JJG, Perez-Silva L, Macias RIR, Asensio M, Peleteiro-Vigil A, Sanchez-Martin A, et al. Molecular Bases of Mechanisms Accounting for Drug Resistance in Gastric Adenocarcinoma. Cancers. 2020;12:2116.

34. De Las Rivas J, Brozovic A, Izraely S, Casas-Pais A, Witz IP, Figueroa A. Cancer drug resistance induced by EMT: novel therapeutic strategies. Arch Toxicol. 2021;95:227997.

35. Zhao M, Liu Y, Qu H. Expression of epithelial-mesenchymal transition-related genes increases with copy number in multiple cancer types. Oncotarget. 2016;7:24688-99.

36. Wee Y, Wang T, Liu Y, Li X, Zhao M. A pan-cancer study of copy number gain and upregulation in human oncogenes. Life Sci. 2018;211:206-14.

37. Zhao M, Zhao Z. Concordance of copy number loss and down-regulation of tumor suppressor genes: a pan-cancer study. BMC Genomics. 2016;17 Suppl 7:532. 
38. Tong Y, Tang Y, Li S, Zhao F, Ying J, Qu Y, et al. Cumulative evidence of relationships between multiple variants in 8q24 region and cancer incidence. Medicine . 2020;99:e20716.

39. Arakawa N, Sugai T, Habano W, Eizuka M, Sugimoto R, Akasaka R, et al. Genomewide analysis of DNA copy number alterations in early and advanced gastric cancers. Mol Carcinog. 2017;56:527-37.

40. Anauate AC, Leal MF, Wisnieski F, Santos LC, Gigek CO, Chen ES, et al. Analysis of 8q24.21 miRNA cluster expression and copy number variation in gastric cancer. Future Med Chem. 2019;11:947-58.

41. Labrador L, Torres K, Camargo M, Santiago L, Valderrama E, Chiurillo MA. Association of common variants on chromosome 8q24 with gastric cancer in Venezuelan patients. Gene. 2015;566:120-4.

42. Jin D-H, Park S-E, Lee J, Kim K-M, Kim S, Kim D-H, et al. Copy Number Gains at 8q24 and 20q11-q13 in Gastric Cancer Are More Common in Intestinal-Type than Diffuse-Type. PLoS One. 2015;10:e0137657.

43. Korivi BR, Faria S, Aly A, Sun J, Patnana M, Jensen CT, et al. Intestinal and diffuse gastric cancer: a retrospective study comparing primary sites. Clin Imaging. 2019;56:3340 .

44. Cancer Genome Atlas Research Network. Comprehensive molecular characterization of gastric adenocarcinoma. Nature. 2014;513:202-9.

45. Qin H, Ni H, Liu Y, Yuan Y, Xi T, Li X, et al. RNA-binding proteins in tumor progression. J Hematol Oncol. BioMed Central; 2020;13:1-23.

46. Morgan MA, Shilatifard A. Chromatin signatures of cancer. Genes Dev. 2015;29:23849.

47. Brabletz T, Kalluri R, Nieto MA, Weinberg RA. EMT in cancer. Nat Rev Cancer. 2018;18:128-34.

48. Bhullar KS, Lagarón NO, McGowan EM, Parmar I, Jha A, Hubbard BP, et al. Kinasetargeted cancer therapies: progress, challenges and future directions. Mol Cancer. 2018;17:48.

49. Olea-Flores M, Zuñiga-Eulogio MD, Mendoza-Catalán MA, Rodríguez-Ruiz HA, Castañeda-Saucedo E, Ortuño-Pineda C, et al. Extracellular-Signal Regulated Kinase: A Central Molecule Driving Epithelial-Mesenchymal Transition in Cancer. Int J Mol Sci. 2019;20:2885.

50. Castel P, Toska E, Engelman JA, Scaltriti M. The present and future of PI3K inhibitors for cancer therapy. Nat Cancer. 2021;2:587-97. 
51. Zhang Y, Kwok-Shing Ng P, Kucherlapati M, Chen F, Liu Y, Tsang YH, et al. A PanCancer Proteogenomic Atlas of PI3K/AKT/mTOR Pathway Alterations. Cancer Cell. 2017;31:820-32.e3.

52. Assumpção PP, Barra WF, Ishak G, Coelho LGV, Coimbra FJF, Freitas HC, et al. The diffuse-type gastric cancer epidemiology enigma. BMC Gastroenterol. 2020;20:223.

53. Sondka Z, Bamford S, Cole CG, Ward SA, Dunham I, Forbes SA. The COSMIC Cancer Gene Census: describing genetic dysfunction across all human cancers. Nat Rev Cancer. 2018;18:696-705.

54. Zhang D, Huo D, Xie H, Wu L, Zhang J, Liu L, et al. CHG: A Systematically Integrated Database of Cancer Hallmark Genes. Front Genet. 2020;11:29.

55. Wan J, Zhou J, Zhao H, Wang M, Wei Z, Gao H, et al. Sonic hedgehog pathway contributes to gastric cancer cell growth and proliferation. Biores Open Access. 2014;3:53-9.

56. Krupar R, Watermann C, Idel C, Ribbat-Idel J, Offermann A, Pasternack H, et al. In silico analysis reveals EP300 as a panCancer inhibitor of anti-tumor immune response via metabolic modulation. Sci Rep. 2020;10:9389.

57. Dong X, Hong Y, Sun H, Chen C, Zhao X, Sun B. NDRG1 suppresses vasculogenic mimicry and tumor aggressiveness in gastric carcinoma. Oncol Lett. 2019;18:3003-16.

58. Liang L, Fang J-Y, Xu J. Gastric cancer and gene copy number variation: emerging cancer drivers for targeted therapy. Oncogene. 2016;35:1475-82.

59. Mariotti S, Barravecchia I, Vindigni C, Pucci A, Balsamo M, Libro R, et al. MICAL2 is a novel human cancer gene controlling mesenchymal to epithelial transition involved in cancer growth and invasion. Oncotarget. 2016;7:1808-25.

60. Herold S, Herkert B, Eilers M. Facilitating replication under stress: an oncogenic function of MYC? Nat Rev Cancer. 2009;9:441-4.

61. Zhang L, Hou Y, Ashktorab H, Gao L, Xu Y, Wu K, et al. The impact of C-MYC gene expression on gastric cancer cell. Mol Cell Biochem. 2010;344:125-35.

62. Huang X, Li Z, Zhang Q, Wang W, Li B, Wang L, et al. Circular RNA AKT3 upregulates PIK3R1 to enhance cisplatin resistance in gastric cancer via miR-198 suppression. Mol Cancer. 2019;18:71. 